\title{
Can We Conquer Advanced Head and Neck Cancer? Current Status and Future Directions
}

\author{
Ho-Ryun Won (ib · Bon Seok Koo (i) \\ Department of Otolaryngology-Head and Neck Surgery, Chungnam National University College of Medicine, Daejeon, Korea
}

Head and neck cancer (HNC) affects approximately 900,000 people annually worldwide [1]. The incidence rate of HNC may appear to be low because it is epidemiologically classified into several subsites (oropharynx, nasopharynx, hypopharynx, larynx, salivary glands and lip, oral cavity), for which separate rates are reported. However, when all subsites are integrated, it corresponds to a high incidence rate that ranks fifth among all cancers in men. In other words, HNC is a commonly encountered disease that should be recognized as important.

In the past decades, many treatment modalities and diagnostic techniques have been developed for HNC. Nevertheless, the mortality rate for oral cavity cancer, a representative type of HNC, continues to increase in males by about $1 \%$. Of course, the overall 5 -year survival rate of $\mathrm{HNC}$ is gradually increasing. However, advanced HNC, such as cases with regional invasion or distant metastasis, excluding tonsil cancer, still presents a poor prognosis with a 5-year survival rate of around $50 \%$, and no remarkable improvement has occurred in recent years [2]. Therefore, in order to improve the outcomes of advanced HNC treatment, it is necessary to move in two directions: (1) improvement and adjustment of existing treatment methods and (2) development of new treatment methods.

Currently, the treatment method of advanced HNC is based on single or combined treatment of surgery, systemic therapy, and radiation therapy (RT). In the recently published National Comprehensive Cancer Network guideline, advanced HNC is classified according to the subsite and an appropriate treatment method is suggested accordingly. However, there is usually no difference in preference regarding the selection of surgery, concurrent systemic therapy/RT, definitive RT, induction chemotherapy, or clinical trials as the initial treatment. This means that the exact effects of each treatment method are ambiguous. In addition, the precise indications for surgery as the initial treatment for advanced HNC are unclear. Lee et al. [3] recently reported the outcomes of several treatment strategies for locally advanced HNC. In addition, Park et al. [4] also reported on the effectiveness and treatment results of surgery for advanced HNC. As such, existing treatment methods should be regularly re-evaluated. It is also important to establish the optimal conditions of the existing treatment methods for advanced HNC through additional prospective studies. In addition, more clinically useful surgical criteria that integrate aspects of functional preservation, including reconstruction, rather than simply stating whether surgery is possible according to $\mathrm{T}$ stage or resectability, will be needed.

Developing new treatment methods for advanced HNC is an important another step towards improving HNC treatment. In recent years, anti-cancer drugs for HNC have been the most actively researched field. Precision medicine has attracted particular attention with the development of targeted therapeutics, and its importance is increasing with the spread of next-generation sequencing. HNC treatment has shown a similar trend. In 2008, cetuximab, a molecular targeted therapeutic agent, was approved for the first time in advanced HNC based on the Erbitux in First-Line Treatment of Recurrent or Metastatic Head and Neck Cancer (EXTREME) study [5]. Currently, cetuximab is recommended for use as the primary regimen in certain circumstances. In addition, as its effects on immune checkpoint inhibitors were confirmed in a phase III trial, it was recently approved for use in recurrent/metastatic HNC. Nivolumab and pembrolizumab, which are anti-programmed cell death-1 (PD-1) antibodies, were approved in 2017 and 2019, respectively, and are used alone or in combination with cytotoxic drugs [6,7]. Clinical studies are continuing to investigate the effects of new immune checkpoint inhibitors, such as ipilimumab and avelumab, on advanced HNC. However, these molecular targeted therapies and immune checkpoint inhibitors have limitations in terms of patient selection, and there are several disadvantages such as resistance to treatment, side effects, and tumor heterogeneity. Therefore, targeted photodynamic therapy or mitochondrial target therapy, which can overcome the shortcomings of existing HNC treat-

Copyright (C) 2021 by Korean Society of Otorhinolaryngology-Head and Neck Surgery.

This is an open-access article distributed under the terms of the Creative Commons Attribution Non-Commercial License (https://creativecommons.org/licenses/by-nc/4.0)

which permits unrestricted non-commercial use, distribution, and reproduction in any medium, provided the original work is properly cited. 
ments, is a field where intriguing results are expected in the future.

The conquest of all cancers, including HNC, is a shared challenge in the medical field that will require major investments of effort and time to solve. In particular, for advanced HNC treatment, since conventional treatments have not yielded a significant increase in the survival rate, further progressive improvements are needed. If existing treatment methods are improved through continuing research, and new treatment methods are applied based on the development of new technologies or drugs, the conquest of advanced HNC will not be too far in the future.

\section{CONFLICT OF INTEREST}

No potential conflict of interest relevant to this article was reported.

\section{ORCID}

Ho-RyunWon https://orcid.org/0000-0002-5135-2474

Bon Seok Koo https://orcid.org/0000-0002-5928-0006

\section{AUTHOR CONTRIBUTIONS}

Conceptualization: BSK. Writing-original draft: HRW. Writingreview \& editing: BSK.

\section{REFERENCES}

1. Sung H, Ferlay J, Siegel RL, Laversanne M, Soerjomataram I, Jemal A, et al. Global cancer statistics 2020: GLOBOCAN estimates of incidence and mortality worldwide for 36 cancers in 185 countries. CA Cancer J Clin. 2021 Feb 4 [Epub]. https://doi.org/10.3322/caac. 21660.

2. Pulte D, Brenner H. Changes in survival in head and neck cancers in the late 20th and early 21st century: a period analysis. Oncologist. 2010;15(9):994-1001.

3. LeeYG, Kang EJ, Keam B, Choi JH, Kim JS, Park KU, et al.Treatment strategy and outcomes in locally advanced head and neck squamous cell carcinoma: a nationwide retrospective cohort study (KCSG HN13-01). BMC Cancer. 2020 Aug;20(1):813.

4. Park JO, Park YM, Jeong WJ, Shin YS, Hong YT, Choi IJ, et al. Survival benefits from surgery for stage IVa head and neck squamous cell carcinoma: a multi-institutional analysis of 1,033 cases. Clin Exp Otorhinolaryngol. 2021 May;14(2):225-34.

5. Rivera F, Garcia-Castano A, Vega N, Vega-Villegas ME, GutierrezSanz L. Cetuximab in metastatic or recurrent head and neck cancer: the EXTREME trial. Expert Rev Anticancer Ther. 2009 Oct;9(10): 1421-8.

6. Harrington KJ, Ferris RL, Blumenschein G Jr, Colevas AD, Fayette J, Licitra L, et al. Nivolumab versus standard, single-agent therapy of investigator's choice in recurrent or metastatic squamous cell carcinoma of the head and neck (CheckMate 141): health-related qualityof-life results from a randomised, phase 3 trial. Lancet Oncol. 2017 Aug;18(8):1104-15.

7. Burtness B, Harrington KJ, Greil R, Soulieres D, Tahara M, de Castro $\mathrm{G} \mathrm{Jr}$, et al. Pembrolizumab alone or with chemotherapy versus cetuximab with chemotherapy for recurrent or metastatic squamous cell carcinoma of the head and neck (KEYNOTE-048): a randomised, open-label, phase 3 study. Lancet. 2019 Nov;394(10212):1915-28. 